capacity approaches closely the more primitive representatives of the Neanderthal group as revealed by the table. I had earlier (1936 b) assumed that there must be some relation between Pithecanthropus and Javanthropus soloensis, the latter resembling the former in several primitive characters. On the other hand, there is no doubt that Javanthropus has many peculiarities in common with Sinanthropus, as recently demonstrated by C. U. Ariëns Kappers (1936) in regard to the endocasts. I should not be surprised if Pithecanthropus at some future date should be found to represent nothing else but a special female type of Javanthropus. Since Javanthropus as a whole represents a very primitive form of Nean- derthal man, the line linking Pithecanthropus and Sinanthropus, respectively through Javanthropus or Neanderthal man, to recent man is continuous.
The fact that there may be certain racial deviations does not matter greatly, since the determining factor does not depend on relatively minute differences but on the main course of human development itself.

\section{IITERATURE.}

Black, Davidson (1931). “On an Adolescent Skull of Sinanthropu pekinensis in Comparison with an Adult of the Same Species and with Other Hominid Skulls Recent and Fossil". Palcontologia Sinica, Ser. $\mathrm{D}, 7$, Fasc. 2 .

Kappers, C. U. Ariëns (1936). "The Endocranial Casts of the Fhringsdorf and Homo soloensis Skulls". J. Anat., 71, 61-76.

Weidenreich, Franz (1935). "The Sinanthropus Population of Weidenreich, Franz (1935). "The Sinanthropus Population of Choukoutien (Locality 1) with a Preliminary Roport
coveries". Bull. Geol. Soc. China, 14, No. 4, 427-468.

Weidenreich, Franz (1936a). "Observations on the Form and Proportions of the Endocranial Casts of Sinanthropus pekinensis, Other Hominids and the Great Apes: a Comparative Study of Brain Size". Paloontologia Sinica, Ser. D, 7 , Jasc. 4.

Weidenreich, Franz (1936b). "Sinanthropus pekinensis and its Position in the Line of Human Evolution". Bull. Peking Nat. Hist., 10, Pt. 4, 281-290.

Weidenreich, Franz (1937). "The New Discoveries of Sinanthropus pekinensis and their Bearing on the Sinanthropus and Pithecanthropus pekinensis and their Bearing on the Sinanthropus and Pithecant
Problems". Bull. Geol. Soc. China, Ting Memorial Volume.

\title{
The Structure of Liquids
}

$\mathrm{T}$ HE Edinburgh discussion of the Faraday Society on September 24-26 on the subject of "The Structure of Liquids and Solutions" was a particularly successful and satisfactory conference. The choice of subject had been very happily timed. The theory of the structure of liquids, which has been in recent years approached from a number of different angles, appears now to have reached a stage in which a larger synthesis is possible, and the bringing together in Edinburgh of many of those who have most notably contributed to these theories has markedly helped towards such a synthesis.

The earlier approach to the theory of liquid structure was almost inevitably an extension of the theory of gases, and the continuity of the two states as established theoretically by Van der Waals seemed to justify this treatment. This approach, however, had led to but little advance in explaining most of the characteristic properties of liquids. A recent tendency, on the other hand, which has taken its origin in the X-ray studies of liquid structure, has been to treat the liquid as more closely analogous to the crystalline solid. This has proved a much more fruitful line of advance for ordinary liquids, that is for liquids at temperatures far removed from their critical temperatures.

The chief difference between liquids and crystals is that in liquids we have irregularity of position and orientation of molecules in the place of the regularity of the crystal. arrangement. This irregularity is limited by the general condition that in liquids the molecules are approximately close-packed. A great advance in the study of liquids is the development of the precise method of defining the molecular or atomic configuration of a liquid under any conditions. This has been achieved by means of the distribution function $g(r)$ of Debye and Prins, which gives the probability

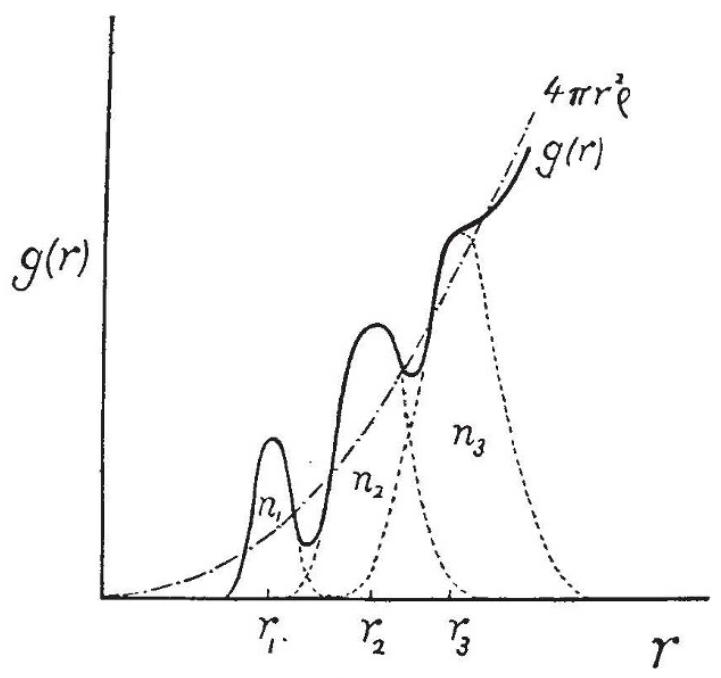

Fig. 1.

of finding a molecule at every distance from an arbitrarily chosen one (see Fig. 1). The peaks of the distribution function correspond to the perfect coordination spheres surrounding atoms in a regular crystal structure, and Prins has suggested that one can be derived from the other by considering each co-ordination sphere of mean radius $r_{k}$ and 
containing $n_{k}$ molecules to be smeared out by a form of error function, so that the distribution function may be written

$$
g(r)=\sum_{k} g_{k}(r)=\sum \frac{n_{k}}{4 \pi r_{k}^{2}} \sqrt{\frac{c}{r_{k}}} e^{-\frac{1}{\lambda^{2}}\left(\frac{r-r_{k}}{k T}\right)^{2}}
$$

Bernal has attempted to apply this idea to the conditions of equilibrium of liquid structures. Any possible configuration of the liquid can be defined by a distribution function depending on only three variables, the average number $N$ of close neighbours of any molecule, the mean distance $R$ of these neighbours, and the variability of this distance, which is equivalent to the measure of the irregularity of the structure. Of all configurations defined in this way, that one will be stable for which each free energy is a minimum for any variations of $N, R$ and $\lambda$. With rise in temperature the values giving such a minimum will change, the co-ordination number in general falling and the irregularity increasing. We may think of the change of configuration of a liquid on heating as equivalent not only to change in the number of excited vibrations, as occurs in a crystal, but more to the transformation of one crystalline phase into another. In a liquid there is an infinite sequence of different configurations, while the condition that the free energy remains constant for any change of phase becomes the general condition of minimum free energy for all configurations. The increasing potential energy on passing from a lowtemperature to a high-temperature configuration consequently gives to liquids a term in the specific heat which does not exist in the crystalline solid, and makes the specific heat of liquids at low temperatures larger than can be explained by any theory based on the excitation of degrees of freedom alone.

Prof. F. London, who presented an admirable introductory paper on "The Nature of the Forces acting between Atoms and Molecules in Liquids", discussed particularly the structure of what should be the ideal, but what is actually the most anomalous of liquids, liquid helium. Here the importance of zero point energy is so great that the minimum of potential energy has little effect in fixing the configuration of the liquid, so that each atom has only four neighbours instead of the twelve that occur in normal liquids. E. Bartholomé and A. Eucken and L. Brillouin dealt with the theory of specific heats of liquids. So far, however, no quantitative theory has been successfully evolved to cover either the behaviour of liquids at very low temperatures or near the critical point, where the general theory indicated above would require considerable modification.

The crucial question of whether the liquid and crystalline states form a continuum in the same sense as liquid and gaseous states was much discussed. J. Frenkel claimed that such a continuum exists, from theoretical considerations, and believes that the transition between crystals and liquids can only be achieved at low temperatures under large negative pressures. F. Simon, on the basis of experimental evidence, considers that, if it occurs at all, it is only at high temperatures and positive pressures. According to Bernal, on the other hand, for purely geometrical reasons no intermediate state should be possible. This question, however, is probably premature, as the theory is not sufficiently advanced and the experimental conditions very difficult to achieve.

Several very notable semi-empirical generalizations on the properties of liquids were given to the conference. According to Bauer and Magat, it appears that a number of properties of liquids, notably their specific volume, surface tension and specific heat, can all be plotted on the same curves, if the temperature ordinate be taken as $\theta=\left(T-T_{F}\right) /\left(T_{C}-T_{F}\right)$, where $T_{C}$ and $T_{F}$ are the critical and melting temperatures respectively. These generalizations would seem to imply that the structures of different liquids resemble one another far more than do the structures of corresponding crystals; they would almost merit the sweeping statement that there is only one liquid state. This requires to be qualified, however, by the possibility that for all the liquids investigated, except water, which is as usual anomalous, the molecules or molecular groups are in a state of free rotation, and that consequently the generalization applies only to assemblages of effectively spherical units. Liquids with highly asymmetrical molecules would tend towards the greater regularity of the liquid crystalline state. Hudleston has shown that similar regularities occur in the compressibilities of liquids, and that they can be referred to a very simple semi-empirical law, while A. G. Ward, by considering the constant $B$ in the viscosity temperature relation,

$$
\eta=A e^{B / R T} \text {, }
$$

has shown that it is closely related to the latent heat of melting and the general physical type of the liquid.

A new method of investigating the properties of liquids, the measurement of absorption of ultrasonic waves studied by M. P. Biquard and J. Errera, has led until now to highly anomalous results. This absorption is found to be larger, and sometimes as as much as a thousand times larger, than that which would be expected under existing theories, and this suggests very forcibly that there are some phenomena in liquids, possibly related to changes of configuration, that have an appreciable characteristic time of the order of a millionth of a second. 
The second part of the conference, devoted to solutions, is more difficult to summarize because here general principles were not so much in evidence. Prof. J. H. Hildebrand, in his introductory paper, laid stress on the need of greater precision in defining the nature of the intermolecular forces in solutions and the meaning of association. In particular he emphasized the much greater importance of the extremely active hydrogen and hydroxyl bonds in liquids compared with the less localized dipole forces.

There was considerable discussion as to the validity of Raoult's law for liquid mixtures and in the changes of entropy on solution, but the general impression, well expressed in Prof. J. 'Kendall's paper, was that in this field anomalies are more apparent than regularities. The structure of liquids themselves being so complex, that of solutions, except dilute solutions of very simple substances, must be far more so. There are so many factors, in the size and shape of the molecules, in the existence of active association centres and in the possibility of formation of limited or indefinite clusters, that it is only in groups of fairly closely related substances in the same solvent that regularities may be expected. Thus, for example, J. A. V. Butler has shown that a series of aliphatic alcohols dissolved in water have heats of solution which can be calculated additively from the number of carbon atoms they contain, and that the entropy of solution is a linear function of the heat of solution.
The value of new methods, such as those of density and magnetic susceptibility, in studying solutions, was brought out in many papers. Perhaps the most important was the study of the infra-red absorption of solutions carried out by J. Errera. He was able to show, for example, by a change of frequency in absorption in a solution of alcohol in carbon tetrachloride, that the alcohol molecules are appreciably associated in pairs at concentrations so low as $0 \cdot 1 \mathrm{~mol}$.

With the analogous use of Raman spectra, it should be possible to determine in liquids and liquid mixtures the degree and kind of molecular association, because whenever this association is a close one it will affect both infra-red and Raman spectra. It may even be possible in some cases to calculate the intermolecular potentials from some form of model structure, as proposed by Magat. But so far, except in the case of water, where quantitative agreement can be got, the results are only qualitative. It is clear, however, that the time has come to begin attack on the problems of liquids and solutions on the basis of molecular theory, and that all this means we shall be able to explain many, at any rate, of the difficulties that have remained unresolved by the application of classical methods in this field.

The Edinburgh discussion of the Faraday Society showed the beginning of this process, and it is to be hoped that if the lead there given is followed up, the structure of liquids will no longer remain the most unsatisfactory part of molecular physics.

\section{Obituary Notices}

\section{Mr. P. A. Ellis Richards}

$\mathrm{B}^{\mathrm{Y}}$ the death on December 22 last of Percy Andrew Ellis Richards at the age of sixty-eight years, Great Britain lost one of its foremost public analysts, chemistry one of its most devoted servants, and the teaching profession one who graced it throughout a long and successful career. Born in 1868, and educated at St. Paul's School and King's College, London, he studied analytical chemistry under the late Prof. C. W. Heaton, whom he succeeded as lecturer in chemistry at Charing Cross Hospital. After the passing of his chief, he set up in practice as a consulting and analytical chemist, and was soon appointed public analyst for the parish of St. Martinin-the-Fields, continuing his work in this capacity when his parish was joined with others to form the City of Westminster. Not long after, he was appointed to a similar post at Hammersmith, and held both until a few years ago, when failing health compelled his retirement.
During his analytical career, Richards carried out work upon the 'facing' of rice, on vinegar, and on preservatives in imported meat for the Local Government Board-now the Ministry of Health. He also served on the Departmental Committee on Preserva. tives and Colouring Matters in Food appointed by the Minister of Health in 1923, and on the Departmental Committee on 'Improvers' in Flour in 1925. He contributed numerous papers to scientific journals upon topics not only in his own sphere, but also upon other subjects such as the saline waters of Boston Spa and of Salsomaggiore, ancient Russian and Irish bog oak, the determination of platinum in alloys, and the determination of iron in animal organs in connexion with researches on pernicious anæmia.

Richards' merits as a teacher were no less than those as an analyst. Very many past students of Charing Cross and the Royal Dental Hospitals and of Queen's College, London, will recall with gratitude his gentle and painstaking manner no less than his 